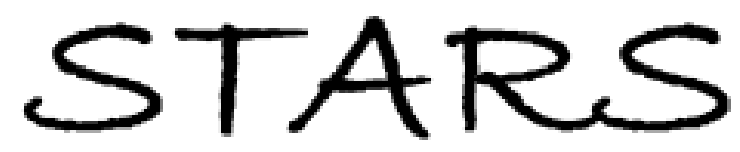

University of Central Florida

STARS

$1-1-2013$

\title{
A generalized electrodynamics model for surface enhanced Raman scattering and enhanced/quenched fluorescence calculations
}

Haining Wang

University of Central Florida

Shengli Zou

University of Central Florida

Find similar works at: https://stars.library.ucf.edu/facultybib2010

University of Central Florida Libraries http://library.ucf.edu

This Article is brought to you for free and open access by the Faculty Bibliography at STARS. It has been accepted for inclusion in Faculty Bibliography 2010 s by an authorized administrator of STARS. For more information, please contact STARS@ucf.edu.

\section{Recommended Citation}

Wang, Haining and Zou, Shengli, "A generalized electrodynamics model for surface enhanced Raman scattering and enhanced/quenched fluorescence calculations" (2013). Faculty Bibliography 2010s. 4823. https://stars.library.ucf.edu/facultybib2010/4823

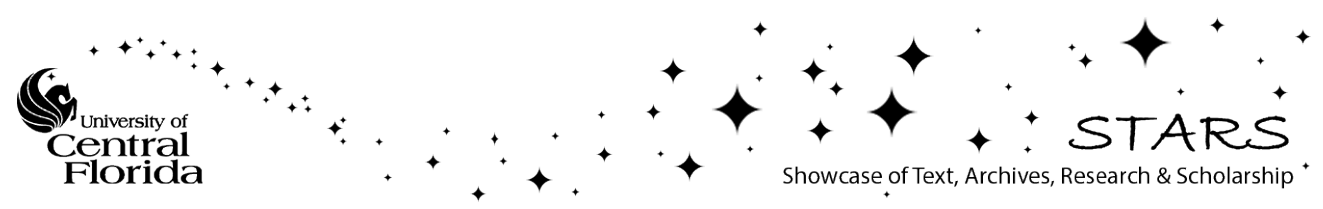




\title{
A generalized electrodynamics model for surface enhanced Raman scattering and enhanced/quenched fluorescence calculations
}

\author{
Haining Wang ${ }^{* a b}$ and Shengli Zou*a \\ A generalized electrodynamics model is proposed to describe the enhancement and quenching of the \\ fluorescence signal of a dye molecule placed near a metal nanoparticle (NP). Both the size of the NP and \\ quantum yield of the dye molecule are crucial in determining the emission intensity of the molecule. \\ Changing the size of the metal NP will alter the ratio of the scattering and absorption cross sections of \\ the metal NP and consequently results in different enhancement or quenching effect to the dye molecule. \\ A dye molecule with a reduced quantum yield indicates that the non-radiative channel is dominant in the \\ decay of the excited dye molecule and the amplification of the radiative decay rate will be easier. In \\ general, the emission intensity will be quenched when the size of the metal NP is small and the quantum \\ yield of the molecule is close to unity. A significant enhancement factor will be obtained when the \\ quantum yield of the molecule is small and the particle size is large. When the quantum yield of the dye \\ molecule is less than $10^{-5}$, the model is simplified to the surface enhanced Raman scattering equation.
}

Received 14th July 2013

Accepted 9th September 2013

DOI: 10.1039/c3ra43603a

www.rsc.org/advances

placed near metal nanoparticles have been reported. Enhanced fluorescence has been experimentally demonstrated by using various types of metallic structures ${ }^{11,12}$ including spherical nanoparticles, ${ }^{2,13-16}$ core-shell nanoparticles, ${ }^{17-22}$ nanorods, ${ }^{23}$ bowtie structures, ${ }^{24}$ or tips. ${ }^{25-27}$ Moerner group reported that an enhancement factor of up to 1340 could be obtained for a single molecule placed between a gold bowtie nanoantenna. ${ }^{24} \mathrm{Chou}$ group showed that an enhancement factor of 2970 and $4.5 \times$ $10^{6}$ for area-average and single-molecule placed at a hot spot could be achieved for ICG dye molecule by a designed diskcoupled dots-on-pillar antenna array structure. ${ }^{28}$ In the meanwhile, fluorescence quenching has also been reported by many groups. ${ }^{29-32}$ Dulkeith et al. demonstrated that the fluorescence signal from a Cy5 dye molecule could be quenched by a gold NP of $6 \mathrm{~nm}$ radius. ${ }^{33}$ Novotny and co-workers showed experimentally a continuous transition from fluorescence enhancement to quenching for a single nile blue molecule placed close to a gold spherical NP. ${ }^{34}$

In this paper, we propose an electrodynamics model to demonstrate that either enhancement or quenching could be obtained for the fluorescence signal of a dye molecule when it is placed adjacent to a metal NP. The model gives a generalized perspective for surface-enhanced Raman scattering and enhanced or quenched fluorescence of various kinds of molecules and metals by electrodynamics method. The size of the NP and the quantum yield of the dye molecule are two crucial parameters for the enhancement or quenching of the fluorescence signal. In the calculations, the molecule and the metal NP were treated as oscillating dipoles and they were described by
${ }^{a}$ Department of Chemistry, University of Central Florida, 4104 Libra Drive, Orlando, FL 32816, USA. E-mail: shengli.zou@ucf.edu; Fax: +1-4078232252; Tel: +14078234123

${ }^{b}$ Beijing Key Laboratory of Bio-inspired Energy Materials and Devices, School of Chemistry and Environment, Beihang University, Beijing 100191, P.R. China. E-mail: hwang@buaa.edu.cn; Fax: +86-10-82339539; Tel: +86-10-82339539 
Lorentz model. The Fourier transform was carried out to obtain the dipole moment and local electric field at frequency domain. The coupled-dipole method was then adopted to calculate the emission (scattering) spectra of the system. The properties of the molecule are similar to those of a Cy3 dye molecule and the properties of the metal NP are close to those of gold in water medium.

\section{Methods}

For an excited molecule, it will decay by two channels, one is called radiative decay which will emit photons and the other is through a non-radiative decay channel in which the energy is converted to thermal energy (phonon energy). The quantum yield, $\eta$, of the molecule is defined by

$$
\eta=\frac{k_{\mathrm{r}}}{k_{\mathrm{r}}+k_{\mathrm{nr}}},
$$

where $k_{\mathrm{r}}$ and $k_{\mathrm{nr}}$ refer to the radiative and non-radiative decay constants of the molecule, respectively. When a metal NP is placed nearby the molecule, the pseudo radiative rate constant of the molecule will be modified which is denoted by $k_{\mathrm{t}}$. We call it pseudo radiative rate constant since part of the energy in the newly defined radiative decay channel $\left(k_{\mathrm{t}}\right)$ will be transferred to the metal NP. A portion of the transferred energy will be absorbed by the metal NP and become its thermal energy and only the rest will be emitted eventually as detectable photons. In this regard, the relative ratio between the scattering and absorption cross sections of the metal NP will determine the percentage of the energy to be converted to thermal energy or photonic energy and plays a crucial role in the quenching or enhancement effect of the metal NP to the dye molecule. We define the altered radiative rate constant of the system including the dye molecule and the metal NP as $k_{\mathrm{r}}^{\prime}$. The modified quantum yield $\eta^{\prime}$ of the system can be expressed as

$$
\eta^{\prime}=\frac{k_{\mathrm{r}}^{\prime}}{k_{\mathrm{t}}+k_{\mathrm{nr}}}=\frac{f_{\mathrm{r}} \times k_{\mathrm{r}}}{f_{\mathrm{t}} \times k_{\mathrm{r}}+k_{\mathrm{nr}}},
$$

where $f_{\mathrm{r}}=k_{\mathrm{r}}^{\prime} / k_{\mathrm{r}}$ is the enhancement factor of the radiative rate constant of the system including the dye molecule and the metal NP relative to that of an isolated dye molecule and $f_{\mathrm{t}}=$ $k_{\mathrm{t}} / k_{\mathrm{r}}$ is the enhancement factor of the pseudo radiative rate constant of the molecule itself in the complex system including the dye molecule and the metal NP in comparison to the radiative rate constant of an isolated dye molecule. Since the total energy is conserved, the number of dye molecule at the excited energy state can be assumed to be the same. The fluorescence signal ratio of the molecule due to the presence of a metal particle at the emission wavelength $q_{\mathrm{em}}$ can be calculated by

$$
q_{\mathrm{em}}=\frac{\eta^{\prime}}{\eta}=\frac{f_{\mathrm{r}} \times\left(k_{\mathrm{r}}+k_{\mathrm{nr}}\right)}{f_{\mathrm{t}} \times k_{\mathrm{r}}+k_{\mathrm{nr}}}=\frac{f_{\mathrm{r}}}{f_{\mathrm{t}} \times \eta+1-\eta} .
$$

In the calculations, $f_{\mathrm{r}}$ is proportional to the enhanced local electric field $|E|^{2}$ of the metal NP at the molecule position at the emission wavelength. ${ }^{35}$ Since the excitation rate of the molecule may also be amplified by a factor of $|E|_{\mathrm{ex}}{ }^{2}$ due to the enhanced local electric field of the metal NP at the excitation wavelength, the detectable fluorescence signal enhancement factor can be calculated by

$$
f=|E|_{\mathrm{ex}}^{2} q_{\mathrm{em}}
$$

We may find that eqn (4) is simplified to $|E|_{\mathrm{ex}}^{2} f_{\mathrm{r}}$ which is the equation to calculate the surface enhanced Raman scattering, ${ }^{35}$ when the quantum yield, $\eta$, of the dye molecule is extremely small. The lifetime ratio of the dye molecule with or without the metal particle can be readily derived as

$$
\frac{\tau^{\prime}}{\tau}=\frac{k_{\mathrm{r}}+k_{\mathrm{nr}}}{k_{\mathrm{t}}+k_{\mathrm{nr}}}=\frac{1}{f_{\mathrm{t}} \times \eta+1-\eta},
$$

where $\tau^{\prime}$ and $\tau$ are the lifetimes of the dye molecule with or without the presence of the nearby metal particle.

In the calculations, the molecule and the metal NP were treated as oscillating dipoles using Lorentz model. The displacement of electrons at different time can be obtained using equation

$$
\omega_{0}^{2} x+\gamma \dot{x}+\ddot{x}=\frac{e E_{\mathrm{loc}}}{m},
$$

where $\omega_{0}$ and $\gamma$ are resonance and damping angular frequency of the dye molecule or metal NP, respectively, $x$ is displacement of electrons at each time, $e$ and $m$ are charge and mass of electrons, respectively, and $E_{\text {loc }}$ denotes the local electric field at the dipole position. For the dye molecule, the $E_{\text {loc }}$ includes the incident light and the scattered light from the metal NP, while the $E_{\text {loc }}$ at the metal NP position only includes the scattered light from the molecule.

The dipole moment, $P$, of the dye molecule or metal NP at each time can be obtained by

$$
P=\rho \operatorname{Vex} / \varepsilon_{0}
$$

where $\rho$ and $V$ are the electron density and volume of the particle, respectively, and $\varepsilon_{0}$ is the vacuum permittivity. The dipole moment and local electric field at frequency domain can be obtained after a Fourier transform. Then the coupled-dipole method $^{36}$ was adopted to calculate the extinction, absorption and scattering cross sections of a single particle or a system including a metal NP and a dye molecule.

$$
\begin{gathered}
C_{\mathrm{ext}}=\frac{4 \pi k}{\left|E_{0}\right|^{2}} \sum_{j=1}^{N} \operatorname{Im}\left(E_{\mathrm{inc}, j}^{*} \cdot P_{j}\right), \\
C_{\mathrm{abs}}=\frac{4 \pi k}{\left|E_{0}\right|^{2}} \sum_{j=1}^{N} \operatorname{Im}\left(P_{j} \cdot E_{\mathrm{loc}, j}^{*}-\frac{2 k^{3}}{3} P_{j} \cdot P_{j}^{*}\right), \\
C_{\mathrm{sca}}=\frac{k^{4}}{\left|E_{0}\right|^{2}} \int \mathrm{d} \Omega\left|\sum_{j=1}^{N}\left[P_{j}-\hat{n}\left(\hat{n} \cdot P_{j}\right)\right] \exp \left(-i k \hat{n} \cdot r_{j}\right)\right|^{2},
\end{gathered}
$$

where $k=2 \pi / \lambda$ is the wave vector at a wavelength of $\lambda, \hat{n}$ is a unit vector in the direction of scattering, and $\mathrm{d} \Omega$ is the element of solid angle, $E_{0}$ is the amplitude of the incident light, $E_{\mathrm{inc}, j}$ and $E_{\text {loc, } j}$ represent the incident and local electric field for particle $j$, respectively. $r_{j}$ and $P_{j}$ denote the coordinate and dipole for particle $j$, respectively. The efficiencies can be calculated by 
dividing the calculated cross sections over the physical area of the particle.

The radiative decay rate constants defined in eqn (1)-(5) are represented using the scattering cross section of the dye molecules or metal NPs. To reduce the numerical errors in the calculations, we calculated the scattering cross section of the whole system using eqn (10), and the scattering cross section of the molecule itself by $C_{\mathrm{ext}}-C_{\mathrm{abs}}$ when a metal particle is placed adjacent to it.

\section{Results and discussion}

The schematic of a dye molecule and a metal NP is shown in Fig. 1. The radius of the metal NP is $r$ and the distance between the dye molecule and the metal nanoparticle surface is defined as $d$. A dye molecule is illuminated by the incident light and the scattered light represents the emission of an excited fluorophore. A metal NP is placed close to the dye molecule which is excited by the emitted (scattered) light from the dye molecule. The coupling between the dye and the metal NP is treated with the coupled dipole method and the dipole excitations of both elements are described by Lorentz model as discussed in the previous paragraph. In the calculations, the excitation wavelength is set at $550 \mathrm{~nm}$, the emission wavelength is fixed at 570 $\mathrm{nm}$, and the quantum yield of the molecule is chosen as 0.16. Those parameters are close to a Cy3 molecule. For the metal particle, we use an electron density of $0.80 \times 10^{28} \mathrm{~m}^{-3}$ and a damping frequency of $5 \times 10^{14} \mathrm{rad}^{-1}$ which is close to a $\mathrm{Au}$ metal NP. The resonance wavelength of the metal NP is set at $540 \mathrm{~nm}$.

Fig. 2(a) shows the fluorescence signal ratio of a dye molecule placed near a metal NP at varying distance in comparison to that of an isolated dye molecule. The results were averaged over different orientations relative to the incident wave vector and polarization directions. When the radius of the metal NP is $10 \mathrm{~nm}$, the fluorescence signal of the dye molecule was quenched because the energy transfer from the dye molecule to the metal NP is dominant by the form of non-radiative decay. Theoretical calculations had been experimentally proved in the previous report. ${ }^{29,37}$ When the distance between the molecule and the metal NP is larger than $50 \mathrm{~nm}$, the effect of the metal NP on the molecule becomes very weak and the signal ratio reaches to 1 . However, when a larger metal NP with a radius of $20 \mathrm{~nm}$ was placed near the molecule, the fluorescence signal of the dye

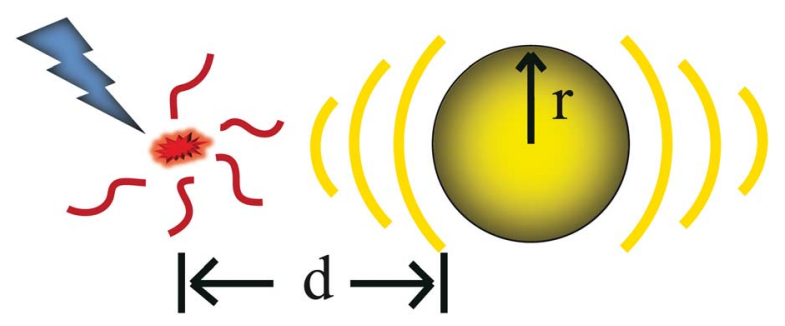

Fig. 1 Schematic of the energy transfer between a dye molecule and a metal particle. $r$ represents the radius of the metal particle and the distance between the dye molecule and the metal particle surface is defined as $d$.
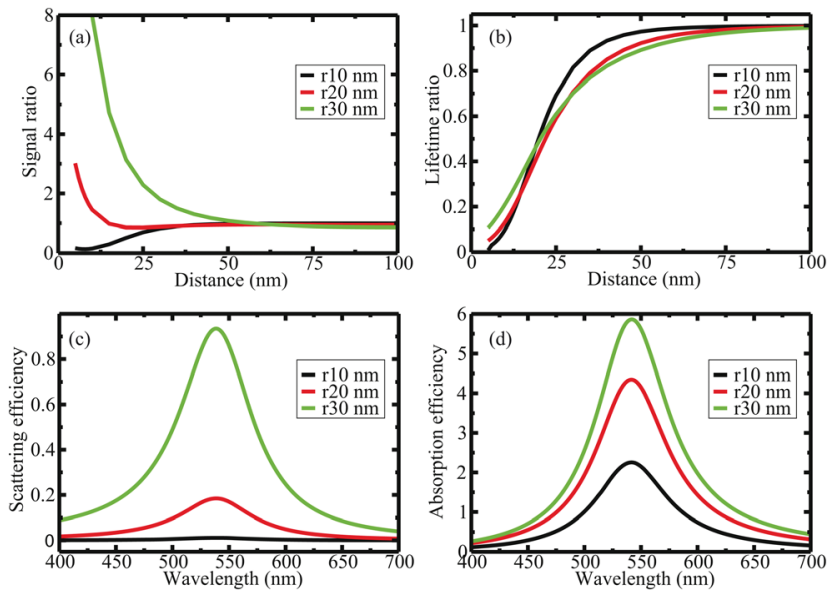

Fig. 2 (a) Fluorescence signal ratio and (b) lifetime ratio of a dye molecule placed near a metal NP with different radii $(r)$ in comparison to those of an isolated dye molecule. (c) The scattering and (d) the absorption spectra of a metal NP with different radii.

molecule is enhanced by a factor of 3 when the metal NP is placed $5 \mathrm{~nm}$ away from the molecule. The enhancement factor reaches to 15 when a metal NP with a $30 \mathrm{~nm}$ radius was used. The alternative enhancement and quenching effect of the metal $\mathrm{NP}$ on the fluorescence signal of the dye molecule can be explained as following. For a metal NP, its scattering cross section is proportional to $r^{6}$ while the absorption cross section is proportional to $r^{3}$ when the radius, $r$, of the particle varies. The different $r$ dependence of the cross sections indicates that the scattering cross section will increase much more significantly than that of the absorption when the radius of the particle is increased. For the presentation clarity of figures, we show scattering and absorption efficiencies in Fig. 2(c) and (d) which are obtained by dividing the corresponding cross sections over $\pi r^{2}$ where $r$ is the radius of the particle. For a small particle of $10 \mathrm{~nm}$ radius as shown in Fig. 2(c) and (d), the scattering efficiency is much smaller in comparison with the absorption efficiency. The scattering efficiency of a metal particle with a $10 \mathrm{~nm}$ radius is only $6.84 \times 10^{-3}$ and the absorption efficiency is 1.48 at emission wavelength of $570 \mathrm{~nm}$. Most of the transferred energy from the dye molecule to the metal NP will be absorbed by the metal NP in the form of nonradiative decay and only small portion is eventually scattered (emitted) as photons, which results in a quenched signal. For a large particle of $30 \mathrm{~nm}$ radius, the scattering efficiency of the metal NP is 0.554 and the absorption efficiency is 3.93 at the emission wavelength. The ratio of the scattering efficiency over the absorption efficiency is much larger than that of a $10 \mathrm{~nm}$ radius metal particle. Consequently, more transferred energy from the excited dye molecule to the metal nanoparticle will decay through the radiative channel and becomes detectable photonic signal which leads to an enhancement of the fluorescence signal.

Fig. 2(b) shows the fluorescence lifetime ratio of a dye molecule placed near a metal NP with different radii at varying distance in comparison to that of an isolated dye molecule. Fig. 2(b) shows that when the distance is short, a small sized 
metal NP results in a shorter lifetime than a larger sized metal NP. When the distance increases, the lifetime ratio of the dye molecule is higher when a metal $\mathrm{NP}$ of $10 \mathrm{~nm}$ radius is placed near the molecule relative to a $30 \mathrm{~nm}$ radius particle. Since the lifetime ratio of the dye molecule as shown in eqn (5) is only related to the ratio of the pseudo radiative decay rate constant of the whole system and the radiative decay constant of an isolated dye molecule $\left(f_{\mathrm{t}}\right)$, Fig. 2(b) shows that the effect of the absorption cross section of the metal NP is more important in altering $f_{\mathrm{t}}$ of the dye molecule than the scattering cross section of the metal NP.

To further understand the proposed mechanism, we numerically change the electron density $\rho$ and damping frequency $\gamma$ of the metal NP to adjust the energy transfer efficiency between the dye molecule and the metal NP. Fig. 3(a) shows the fluorescence signal ratio of a dye molecule placed near a metal NP in comparison to that of an isolated dye molecule when the electron density of the metal NP is changed from $0.8 \times 10^{28}$ to $1.6 \times 10^{28}$ and $3.2 \times 10^{28} \mathrm{~m}^{-3}$. Fig. 3(a) shows that the fluorescence signal is enhanced with a higher factor with increasing electron density of the metal NP. A larger electron density means more electrons in the particle which has a similar effect as increasing particle radius, which results in a larger scattering cross section of the metal nanoparticle. In another hand, a decreased damping frequency leads to reduced absorption cross section. When the damping frequency is decreased from $5 \times 10^{14}$ to $4 \times 10^{14}$ or $3 \times 10^{14} \mathrm{rad}^{-1}$, the ratio between the scattering and absorption cross sections of the metal particle will increase, which will also give a more enhanced fluorescence signal, as shown in Fig. 3(b).

We have showed the fluorescence signal ratio of a dye molecule placed near a metal NP at different conditions. eqn (3) shows that the quantum yield $\eta$ of the molecule is also crucial in calculating the quenching/enhancement factor of the dye molecule placed near a metal NP. From eqn (3) we can find that, when $\eta$ is close to $1(\log (\eta)=0)$, the equation will be reduced to $f_{\mathrm{r}} / f_{\mathrm{t}}$. Since $f_{\mathrm{r}}$ represents the radiative decay rate constant (total emission) enhancement while $f_{\mathrm{t}}$ includes both the emitted energy and the absorbed energy by the metal NP and is always larger than $f_{\mathrm{r}}$, the fluorescence will always be quenched if we did not consider the excitation rate enhancement at the excitation wavelength. In another hand, if $\eta$ is extremely small, eqn (3) is simplified to $f_{\mathrm{r}}$, which is proportional to the enhanced local electric field $|E|^{2}$ of the metal NP
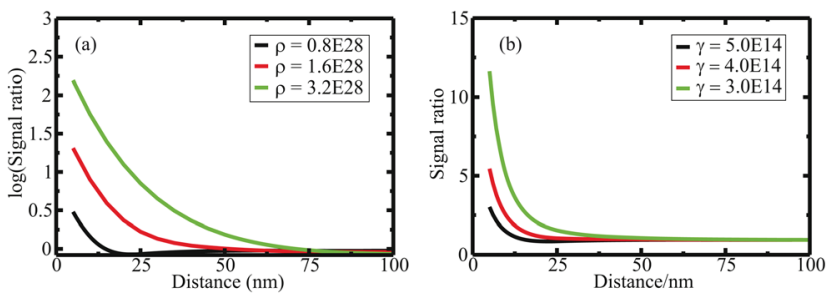

Fig. 3 Fluorescence signal ratio of a dye molecule placed near a metal NP with (a) different electron density $\rho$ or (b) damping frequency $\gamma$.

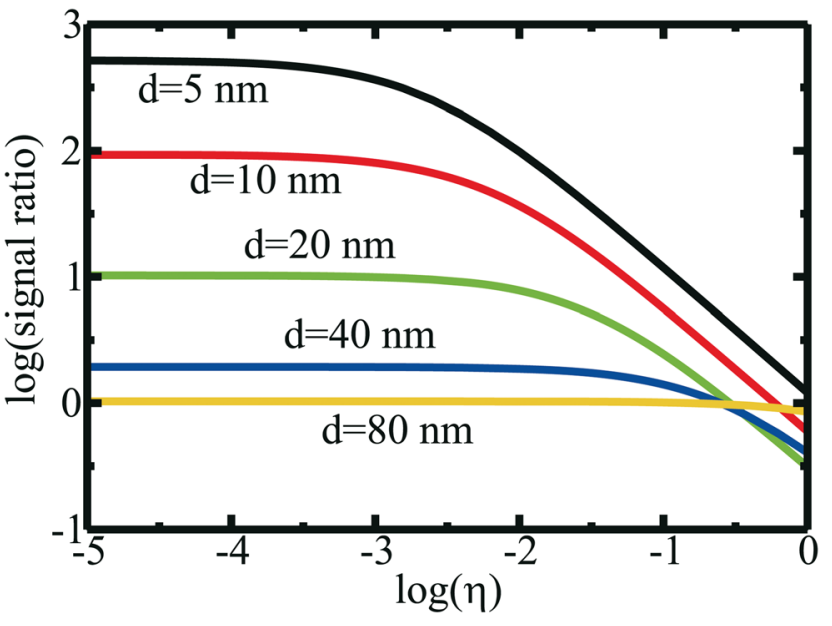

Fig. 4 Fluorescence signal ratio of a dye molecule with varying quantum yield $(\eta)$ placed near a metal NP. The radius of the particle is $20 \mathrm{~nm}$ and the molecule is placed at different distance $(d)$ from the particle surface.

at the emission wavelength at the position of the molecule. eqn (4) is simplified to the equation used to calculate the surface-enhanced Raman scattering. It is worth noting that the enhancement of the Raman signal at emission wavelength is due to the change of the relative decay rate constants between the radiative and non-radiative channels which does not violate the energy conservation of the system. As shown in Fig. 4, when a metal NP with a $20 \mathrm{~nm}$ radius is placed $5 \mathrm{~nm}$ away from the molecule, the signal ratio is enhanced by a factor of 516 if the quantum yield $\eta$ is set at $1 \times 10^{-5}$. Similar results will be obtained when the distance is changed to 10 , 20 , and $40 \mathrm{~nm}$. When the distance is further extended to 80 $\mathrm{nm}$, the enhancement/quenching factor will be close to 1 no matter of the quantum yield of the molecule.

\section{Conclusions}

In conclusion, we proposed a generalized electrodynamics model to calculate the energy transfer efficiency between a dye molecule and a metal NP. When the size of the particle is small (10 $\mathrm{nm}$ radius), the transferred energy from the dye molecule to the metal NP is dominantly absorbed by the metal particle which results in a quenched signal. However, the fluorescence signal will be amplified when a large metal NP $(30 \mathrm{~nm}$ radius or larger) is used. The reason of the change is due to the relative ratio variation of the scattering and absorption cross sections of the metal NP. Increasing the electron density or decreasing the damping frequency of the metal NP will also generate the same effect due to the increased ratio between the scattering and absorption cross sections of the metal NP. The quantum yield of the molecule also has a significant effect on the enhanced or quenched signal. A molecule with a quantum yield close to one will experience a quenched fluorescence signal when placed near a small metal NP in comparison to an isolated molecule. When the quantum yield of the molecule is extremely small, the signal enhancement will be similar to the surface-enhanced Raman scattering. 


\section{Acknowledgements}

We are thankful for the support from the National Science Foundation (NSF) and the Office of Naval Research (ONR) Fund.

\section{Notes and references}

1 C. Blum, N. Zijlstra, A. Lagendijk, M. Wubs, A. P. Mosk, V. Subramaniam and W. L. Vos, Phys. Rev. Lett., 2012, 109, 203601.

2 G. P. Acuna, F. M. Möller, P. Holzmeister, S. Beater, B. Lalkens and P. Tinnefeld, Science, 2012, 338, 506-510.

3 D. J. Maxwell, J. R. Taylor and S. Nie, J. Am. Chem. Soc., 2002, 124, 9606-9612.

4 K. M. Mayer and J. H. Hafner, Chem. Rev., 2011, 111, 38283857.

5 A. Haes, D. Stuart, S. Nie and R. Van Duyne, J. Fluoresc., 2004, 14, 355-367.

6 T. Förster, Ann. Phys., 1948, 437, 55-75.

7 T. Dewey and G. Hammes, Biophys. J., 1980, 32, 1023-1035.

8 B. Persson and N. Lang, Phys. Rev. B: Condens. Matter Mater. Phys., 1982, 26, 5409-5415.

9 D. A. Weitz, S. Garoff, J. I. Gersten and A. Nitzan, J. Chem. Phys., 1983, 78, 5324-5338.

10 J. R. Lakowicz, Anal. Biochem., 2005, 337, 171-194.

11 F. Emmanuel and G. Samuel, J. Phys. D: Appl. Phys., 2008, 41, 013001.

12 J. Lakowicz, C. Geddes, I. Gryczynski, J. Malicka, Z. Gryczynski, K. Aslan, J. Lukomska, E. Matveeva, J. Zhang, R. Badugu and J. Huang, J. Fluoresc., 2004, 14, 425-441.

13 Y. Chen, K. Munechika and D. S. Ginger, Nano Lett., 2007, 7, 690-696.

14 R. M. Bakker, H.-K. Yuan, Z. Liu, V. P. Drachev, A. V. Kildishev, V. M. Shalaev, R. H. Pedersen, S. Gresillon and A. Boltasseva, Appl. Phys. Lett., 2008, 92, 043101.

15 A. Bek, R. Jansen, M. Ringler, S. Mayilo, T. A. Klar and J. Feldmann, Nano Lett., 2008, 8, 485-490.

16 S. Eustis and M. A. El-Sayed, Chem. Soc. Rev., 2006, 35, 209217.

17 C. Li, Y. Zhu, X. Zhang, X. Yang and C. Li, RSC Adv., 2012, 2, 1765-1768.

18 P. Du, Y. Cao, D. Li, Z. Liu, X. Kong and Z. Sun, RSC Adv., 2013, 3, 6016-6021.
19 K. T. Shimizu, W. K. Woo, B. R. Fisher, H. J. Eisler and M. G. Bawendi, Phys. Rev. Lett., 2002, 89, 117401.

20 R. Bardhan, N. K. Grady, J. R. Cole, A. Joshi and N. J. Halas, ACS Nano, 2009, 3, 744-752.

21 O. G. Tovmachenko, C. Graf, D. J. van den Heuvel, A. van Blaaderen and H. C. Gerritsen, Adv. Mater., 2006, 18, 91-95.

22 K. Aslan, M. Wu, J. R. Lakowicz and C. D. Geddes, J. Am. Chem. Soc., 2007, 129, 1524-1525.

23 Y. Fu, J. Zhang and J. R. Lakowicz, J. Am. Chem. Soc., 2010, 132, 5540-5541.

24 A. Kinkhabwala, Z. Yu, S. Fan, Y. Avlasevich, K. Mullen and W. E. Moerner, Nat. Photonics, 2009, 3, 654-657.

25 A. Kramer, W. Trabesinger, B. Hecht and U. P. Wild, Appl. Phys. Lett., 2002, 80, 1652-1654.

26 M. Thomas, J.-J. Greffet, R. Carminati and J. R. AriasGonzalez, Appl. Phys. Lett., 2004, 85, 3863-3865.

27 J. M. Gerton, L. A. Wade, G. A. Lessard, Z. Ma and S. R. Quake, Phys. Rev. Lett., 2004, 93, 180801.

28 W. Zhang, F. Ding, W.-D. Li, Y. Wang, J. Hu and S. Y. Chou, Nanotechnology, 2012, 23, 225301.

29 R. Chhabra, J. Sharma, H. Wang, S. Zou, S. Lin, H. Yan, S. Lindsay and Y. Liu, Nanotechnology, 2009, 20, 485201.

30 F. Cannone, G. Chirico, A. R. Bizzarri and S. Cannistraro, J. Phys. Chem. B, 2006, 110, 16491-16498.

31 E. Dulkeith, A. C. Morteani, T. Niedereichholz, T. A. Klar, J. Feldmann, S. A. Levi, F. C. J. M. van Veggel, D. N. Reinhoudt, M. Möller and D. I. Gittins, Phys. Rev. Lett., 2002, 89, 203002.

32 G. P. Acuna, M. Bucher, I. H. Stein, C. Steinhauer, A. Kuzyk, P. Holzmeister, R. Schreiber, A. Moroz, F. D. Stefani and T. Liedl, ACS Nano, 2012, 6, 3189-3195.

33 E. Dulkeith, M. Ringler, T. A. Klar, J. Feldmann, A. Muñoz Javier and W. J. Parak, Nano Lett., 2005, 5, 585-589.

34 P. Anger, P. Bharadwaj and L. Novotny, Phys. Rev. Lett., 2006, 96, 113002.

35 M. Kerker, D.-S. Wang and H. Chew, Appl. Opt., 1980, 19, 4159-4174.

36 S. Zou, N. Janel and G. C. Schatz, J. Chem. Phys., 2004, 120, 10871-10875.

37 S. Pal, P. Dutta, H. Wang, Z. Deng, S. Zou, H. Yan and Y. Liu, J. Phys. Chem. C, 2013, 117, 12735-12744. 